\title{
CCD astrometric observations of Amalthea and Thebe ${ }^{\star, \star \star}$
}

\author{
C. H. Veiga ${ }^{1}$ and R. Vieira Martins ${ }^{1,2,3}$ \\ 1 Observatório Nacional, Rua General José Cristino 77, 20921-400 Rio de Janeiro, Brazil \\ e-mail: cave@on.br \\ 2 Observatório do Valongo, Ladeira Pedro Antônio 43, 20080-090 Rio de Janeiro, Brazil \\ 3 Institut de Mécanique Céleste et de Calcul des Éphémérides, 77 avenue Denfert Rochereau, 75014 Paris, France
}

Received 4 January 2005 / Accepted 14 March 2005

\begin{abstract}
This paper presents the results of observations of Jupiter's satellites Amalthea and Thebe made in 1995, 1996 and 2001 at the Laboratório Nacional de Astrofísica (LNA), Brazil. The observations were made in visible light wavelengths with a $1.6 \mathrm{~m}$ reflector telescope and the light of Jupiter was covered by a mask placed near the CCD surface. The already published positions for 1995, whose astrometric reduction used the Galilean satellites, are now reduced using the stars in the CCD fields like the new positions of 1996 and 2001. The 2001 data are much better than those obtained in 1995, and that those from 1996 show large residuals. Considering the 310 frames observed, the mean residual is about 0 o' $^{\prime} 01$ and the standard deviation is about 0 .' 15 .
\end{abstract}

Key words. astrometry - Jupiter satellites - Amalthea and Thebe

\section{Introduction}

The inner Jovian satellites Amalthea and Thebe whose visual magnitude are 14.1 and 15.7, orbit around the planet at distances of about 2.5 and 3.1 Jupiter radi and so, very close to the bright planet whose magnitude is about -2 . In spite of Amalthea and Thebe proximity to the planet and their relative faintness, some astrometric observations have been made from Earth. Amalthea was discovered by Barnard (1982) and since then many visual, photographic and CCD observations of this satellite, as well as spacecraft and HST images have been made. Thebe was discovered in 1979 when the spacecraft Voyager 1 imaged the inner system of Jupiter (Stone \& Synnott 1980; Synnott 1980) and there are now CCD, spacecraft and HST observations. A review of the published observations of these two satellites can be found in Vachier (2004).

The LNA program of systematic astrometric observations of planetary satellites started in 1982 at the LNA (Laboratório Nacional de Astrofísica, in Brazil). 122 positions of Amalthea and 71 positions of Thebe were published in Veiga \& Vieira Martins (1996). However the astrometric reduction of these observations were made using the positions of the Galilean satellites whose images are always saturated in the CCD frame and so give a poor center determination. Now, results for Amalthea are presented for 93 frames from those earlier observations,

^ Based on observations made at Laboratório Nacional de Astrofísica/MCT-Itajubá-Brazil.

$\star \star$ Table 2 is only available in electronic form at the CDS via anonymous ftp to cdsarc.u-strasbg.fr $(130.79 .128 .5)$ or via http://cdsweb.u-strasbg.fr/cgi-bin/qcat?J/A+A/437/1147 with the reduction made from the stars in the CCD field. Only the positions with residuals smaller than $11^{\prime \prime} 0$ are presented. Also from the earlier data, 80 positions are obtained for Thebe. The new observations are: 26 positions of Thebe referred to Amalthea (hereafter Amalhea-Thebe) made in 1996 and 191 positions for Amalthea and 188 positions for Thebe made in 2001. In Sect. 2, the observations and the reduction are described. The comparison between the observational and the theoretical positions are discussed in Sect. 3. The conclusions are given in Sect. 4.

\section{Observations and reductions}

All the observations were made at the Cassegrain focus of the $1.6 \mathrm{~m}$ Ritchey-Chretien reflector of the LNA (IAU number 874 , geographical longitude: $3^{\mathrm{h}} 02^{\mathrm{m}} 19^{\mathrm{s}}$, latitude: $-22^{\circ} 32^{\prime} 04^{\prime \prime}$, altitude: $1872 \mathrm{~m}$ ). The focal length of the Cassegrain combination is $15.8 \mathrm{~m}$, with a plate scale of $13^{\prime \prime} / \mathrm{mm}$ on the focal plane. The image of the planet is hidden by a round mask on the CCD window near the CCD surface. To avoid diffraction spikes from the secondary mirror bearing structure at the telescope focal plane, an external, eight hole mask is placed on the entrance pupil. The exposure times varied between 10 and $60 \mathrm{~s}$, depending on the CCD sensitivity and on the meteorological conditions. No filter was used. The details of the observations are given in Table 1.

A total of 310 frames was obtained over 8 nights, distributed as follows: 93 frames over 3 nights in 1995, 26 frames over 2 nights in 1996, and 191 frames over 3 nights in 2001. About one hundred frames obtained over 5 nights in 1996 
Table 1. Observational information for each night. The mean exposition time is given in column $T$, in seconds. $N$ is the number of obtained frames. $K$ gives the number of reference stars used in the astrometric calibration of the frames. The dimension of the CCD in pixel is given in column CCD and the length of side of the square pixels in micrometer are in column Pixel. For each frame and for the directions $x$ and $y$, the astrometric calibration gives a standard deviation of the reference star residuals. The column $\sigma$ give the mean of theses standard deviations over the frames of the corresponding night in arseconds.

\begin{tabular}{lccccccc}
\hline \hline Night & $T$ & $N$ & $K$ & CCD & Pixel & \multicolumn{2}{c}{$\sigma$} \\
\hline $95-05-23$ & 10 & 50 & 11 & $1152 \times 770$ & $22.5 \times 22.5$ & 0.1 & 0.2 \\
$95-08-07$ & 60 & 37 & 22 & $1152 \times 770$ & $22.5 \times 22.5$ & 0.2 & 0.2 \\
$95-09-14$ & 60 & 6 & 9 & $1152 \times 770$ & $22.5 \times 22.5$ & 0.1 & 0.1 \\
$96-06-21$ & 20 & 16 & 13 & $1024 \times 1024$ & $24 \times 24$ & 0.2 & 0.2 \\
$96-08-22$ & 30 & 10 & 6 & $1152 \times 770$ & $22.5 \times 22.5$ & 0.3 & 0.2 \\
$01-10-25$ & 12 & 58 & 17 & $1024 \times 1024$ & $24 \times 24$ & 0.2 & 0.2 \\
$01-10-26$ & 15 & 34 & 22 & $1024 \times 1024$ & $24 \times 24$ & 0.1 & 0.2 \\
$01-10-27$ & 16 & 99 & 20 & $1024 \times 1024$ & $24 \times 24$ & 0.1 & 0.2 \\
\hline
\end{tabular}

were not considered due to the small number of reference stars within the frame and also to the poor quality images of the inner satellites.

All frames were measured with the ASTROL software (Colas 1996), which uses a centering algorithm based on a point spread function fit. The local sky background was removed by ASTROL, using a second order polynomial. This background removal is essential for avoiding systematic errors in the measurements of the center of the satellites. The center of Jupiter cannot be measured since the mask is over the image of the planet. For the stars the centering errors are at the level of 0.1 pixel ( $0 .^{\prime} 03$ in telescope plate scale). For Amalthea and Thebe they are at the level of $0.05\left(0 .^{\prime} 02\right)$ and $0.15\left(0 .^{\prime} 05\right)$ respectively.

The equatorial coordinates of the field stars were obtained from the USNO-A2.0 catalog, as locally corrected by UCAC2 positions. For each frame, the local correction consists shift of all the stars of USNO-A2.0, in a field with $2^{\circ} \times 2^{\circ}$ centered on the frame, by the mean of the differences between their positions and those of the UCAC2 catalog. Therefore, the coordinate origins of the corrected positions of all stars in the frame coincide for the two catalogs.

For the astrometric calibration of the observed field, a first degree polynomial is used to map the tangential plane projection of the sky onto the frame. The standard deviation of the residuals for the stars effectively employed in the reduction was at 0.2 and the mean number of reference stars by frame varied from 6 to 25. The values for each night are given in Table 1 . The scales and directions derived from the first degree polynomial are essentially the same for each observational night. However they change for different nights since the CCD positions slightly changed due to maintenance operations.

For 10 frames taken in 1996 the number of measurable catalog stars was not sufficient to obtain good equatorial positions of the satellites. However, the differences of the equatorial coordinates of Amalthea and Thebe present fairly good values. So, for these frames only the positions of Amalthea-Thebe are

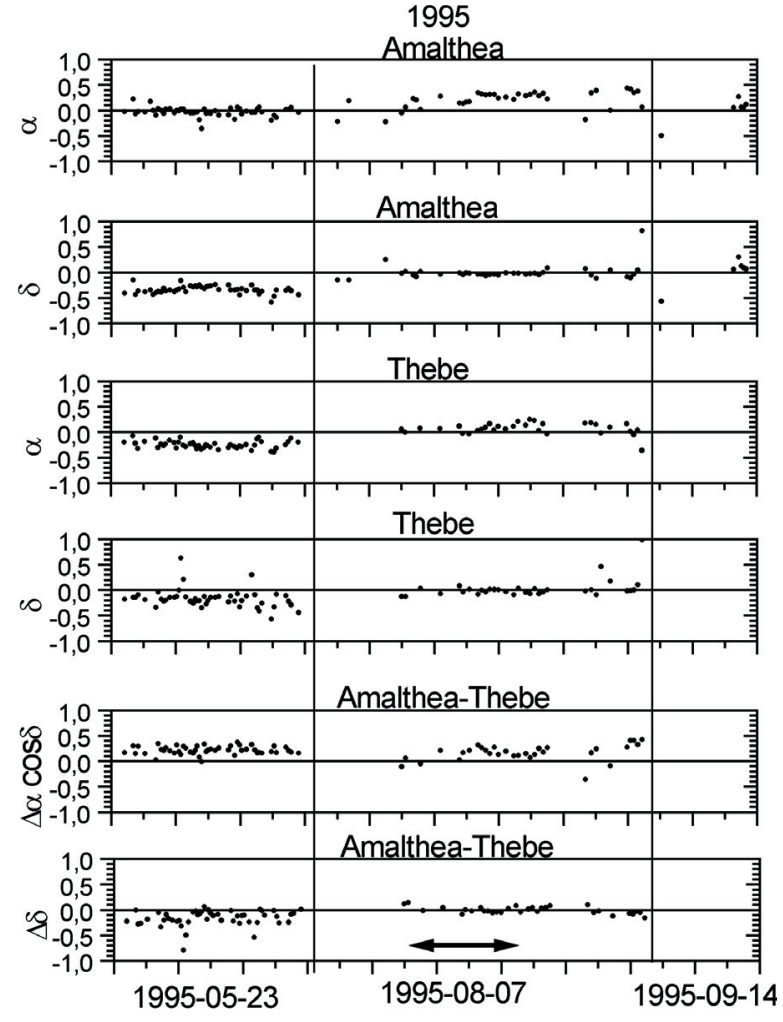

Fig. 1. Residuals of Amalthea, Thebe and Amalthea-Thebe observed positions made in 1995 compared with the JPL ephemerides. Each column corresponds to the observation made on one night, whose date is given at the bottom of the figure. In the $x$ axis, the length corresponding to an hour is given by the double arrows at the bottom.

given. For the other 16 frames also obtained in 1996, the right ascension and the declination of the satellites presented large shifts but the relative positions do not. So, only the relative positions of the satellites are given.

In Table 2, available only in electronic form at the CDS, the topocentric observed positions of Amalthea and Thebe are presented. The reference system is J2000, the instants of observation are given in universal time and presented in Julian days and fractions. The right ascension and declination for both satellites and the $x$ and $y$ coordinates for Amalthea-Thebe have given. In Col. 1 of Table 2 is given the instant of observation in Julian date and fractions, in Cols. 2-4 the Amalthea observed right ascension in hours, minutes and seconds, in Cols. 5-7 the declination of Amalthea in degrees, arcminutes and arcseconds, in Cols. 8-13 the same coordinates for Thebe and in Cols. 14 and $15 x=\Delta \alpha \cos \delta$, and $y=\Delta \delta$ in $\operatorname{arcsec}$.

\section{Comparison with the theoretical positions}

The observations were compared with the JPL ephemeris and the results are presented in Figs. 1-3 and in Table 3. Except for the third night in 1995, all the observations of Amalthea were made around the maximal elongation distance and just before or after the corresponding points for Thebe.

For the first observed night in 1995 (1995-05-23), for Amalthea positions in the south-east elongation and for Thebe in the north-west elongation, the residuals on delta for the 

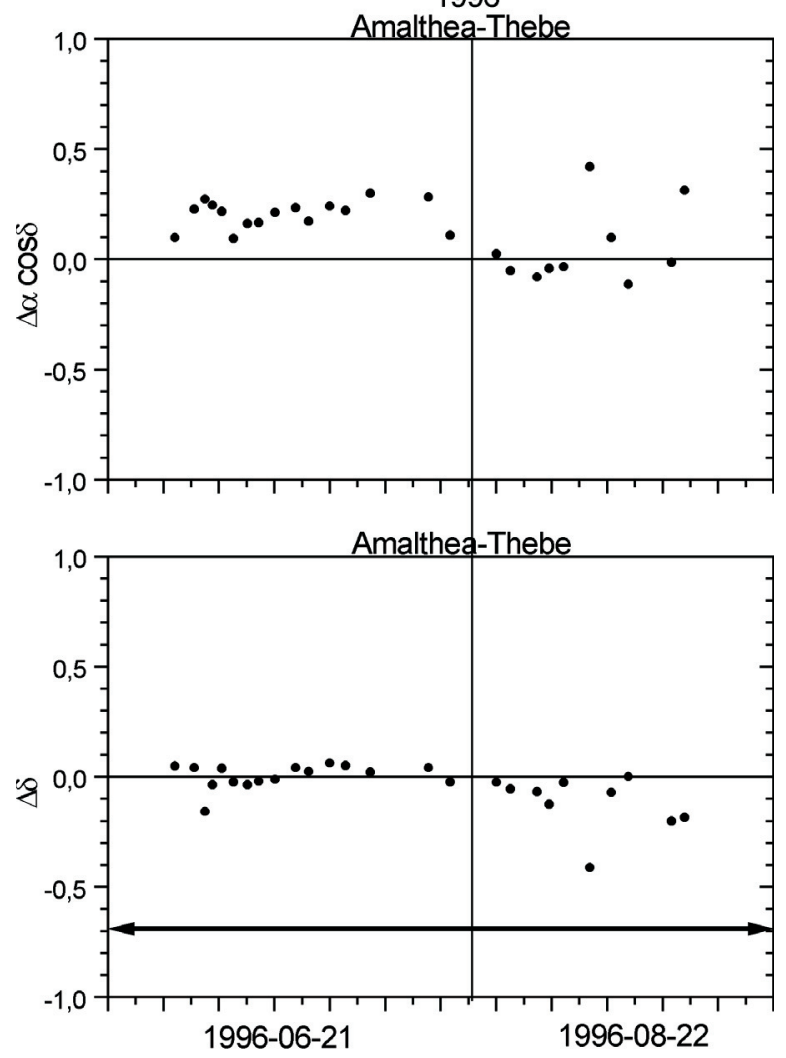

Fig. 2. The same as Fig. 1 but for 1996.

former satellite and on alpha and delta for the second are shifted by about 0.2 . One of the reasons for these large residuals probably is the small number of reference stars used in the reduction (see Table 1) which gives poor astrometric calibration. These residuals are likely not due to an important shift in the equatorial coordinates of the satellites since the Amalthea-Thebe relative residuals also present large shifts.

For the second observed night in 1995 (1995-08-07), when Amalthea and Thebe elongation is north-west, the alpha residuals of Amalthea are shifted by about 0.2 . In this case the number of stars used in the reduction is not small and there is no shift in the equatorial coordinates. The large dispersion on the alpha residuals for both satellites can be explained by the difficulty of measuring the $x$ position of the satellite images contaminated by the light of the planet.

For the last observed night of 1995 (1995-09-14) there is a small number of observed frames showing Amalthea and the astrometric reduction was made with a few stars. Therefore it is difficult to analyze the shift of $0 . ' 1$ obtained for the residuals in alpha and delta.

In the two nights in 1996 the alpha and delta residuals of the satellites presented a very large shift. Therefore, only their relative positions were considered. For the second night (1996-08-22) this shift can be due to the small number of reference stars used in the reduction. However, for the first night (1996-06-21) no special reason was found to justify this fact, in spite of the different procedures used for astrometric reduction.

The three nights observed in 2001 present the best results of our observations of Amalthea and Thebe. All the Amalthea

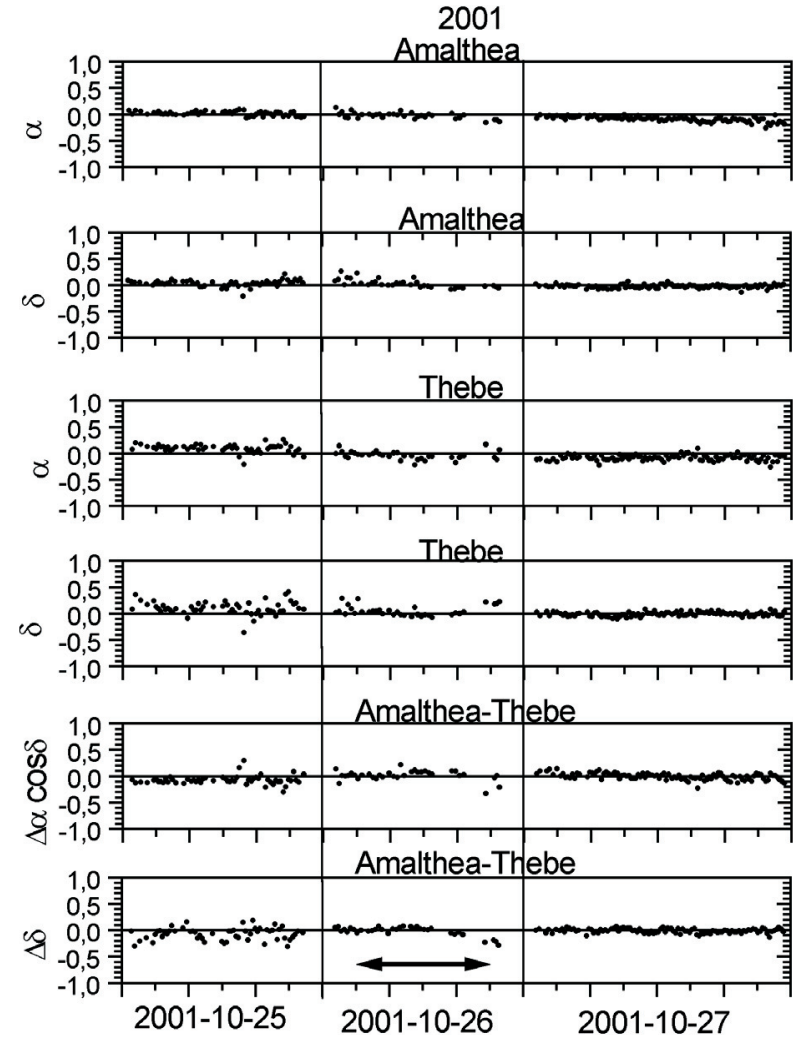

Fig. 3. The same as Fig. 1 but for 2001.

Table 3. a) Observed minus calculated statistics of Amalthea for each night. The units are arcseconds and $\bar{\alpha}, \sigma_{\alpha}, \bar{\delta}, \sigma_{\delta}$ are the means and the standard deviations for the residuals in right ascension and declination. $N$ is the number of observations. In the first column the date of the observation is given and in the last line the statistics for all the observations.

\begin{tabular}{lccccr}
\hline \hline Date & $\bar{\alpha}$ & $\sigma_{\alpha}$ & $\bar{\delta}$ & $\sigma_{\delta}$ & $N$ \\
\hline $95-05-23$ & -0.029 & 0.090 & -0.337 & 0.076 & 50 \\
$95-08-07$ & 0.209 & 0.172 & 0.001 & 0.155 & 37 \\
$95-09-14$ & 0.116 & 0.081 & 0.122 & 0.094 & 6 \\
$01-10-25$ & 0.015 & 0.039 & 0.033 & 0.060 & 58 \\
$01-10-26$ & -0.027 & 0.062 & 0.030 & 0.082 & 34 \\
$01-10-27$ & -0.093 & 0.048 & -0.027 & 0.033 & 99 \\
All & -0.010 & 0.130 & -0.058 & 0.158 & 284 \\
\hline
\end{tabular}

positions were observed around the north-east maximal elongation and the residual dispersion is very small, considering the difficulty involved in measuring and reducing the frames. A relatively large shift of $-0 .{ }^{\prime} 09$ on the alpha residual can be observed for the third night (2001-10-27), but it exists also for the observations of Thebe on the same night. This suggests that the right ascension positions were calculated with a shift for this reference field. All Thebe positions were observed near the maximal elongation. For the first and the last nights (2001-1025 and 2001-10-27), Thebe was near the south-west elongation and for the second night (2001-10-26), in the north-east elongation. Its residuals present a larger dispersion than Amalthea's ones. This is due to the dim magnitude of this satellite which 
Table 3. b) The same as Table 3a for Thebe.

\begin{tabular}{lccccr}
\hline \hline Date & $\bar{\alpha}$ & $\sigma_{\alpha}$ & $\bar{\delta}$ & $\sigma_{\delta}$ & $N$ \\
\hline 95-05-23 & -0.247 & 0.076 & -0.169 & 0.184 & 48 \\
$95-08-07$ & 0.071 & 0.113 & 0.029 & 0.203 & 32 \\
$01-10-25$ & 0.091 & 0.077 & 0.102 & 0.127 & 55 \\
$01-10-26$ & -0.042 & 0.080 & 0.051 & 0.098 & 34 \\
$01-10-27$ & -0.086 & 0.055 & -0.012 & 0.040 & 99 \\
All & -0.054 & 0.137 & -0.004 & 0.153 & 268 \\
\hline
\end{tabular}

Table 3. c) The same as Table 3 a for Amalthea minus Thebe.

\begin{tabular}{lccccr}
\hline \hline Date & $\bar{x}$ & $\sigma_{x}$ & $\bar{y}$ & $\sigma_{y}$ & $N$ \\
\hline $95-05-23$ & 0.213 & 0.080 & -0.166 & 0.156 & 48 \\
$95-08-07$ & 0.164 & 0.163 & -0.008 & 0.069 & 31 \\
$96-06-21$ & 0.202 & 0.064 & 0.003 & 0.055 & 16 \\
$96-08-22$ & 0.051 & 0.177 & -0.117 & 0.124 & 10 \\
$01-10-25$ & -0.074 & 0.085 & -0.073 & 0.117 & 55 \\
$01-10-26$ & 0.015 & 0.097 & -0.022 & 0.091 & 34 \\
$01-10-27$ & -0.006 & 0.063 & -0.015 & 0.041 & 99 \\
All & 0.051 & 0.141 & -0.053 & 0.111 & 293 \\
\hline
\end{tabular}

makes its centering dificult. For the first night the alpha and the delta residuals are shifted by about 0 .' 1 . Comparing these residuals with those obtained from the relative positions of Amalthea-Thebe, no reduction feature seems to explain these shifts.

\section{Conclusion}

The results presented here for the observations made in 2001 present residuals as good as the best in the literature. Since the ground-based observations of Amalthea and Thebe and their reductions are very difficult, there is only a small number of published observations and most are of lower quality. However, recently Kulyk et al. (2002) published an ensemble of high standard CCD observations with 35 positions for Amalthea and 28 for Thebe observed on
4 nights in 1999 and 2000. The mean of the residuals (and root mean square in brackets) of their 25 observations of Amalthea in 1999 are respectively $0.03\left(0 .{ }^{\prime} 10\right)$ for alpha and $-0 .{ }^{\prime} 06$ $\left(0{ }^{\prime} 05\right)$ for delta, and for the 10 observations in 2000 the mean of the residuals are -0 . $^{\prime} 07(0.22)$ and $-0 .{ }^{\prime} 06(0 . ' 18)$ respectively for alpha and delta. For the 28 frames of Thebe made in 1999, they are $-0 . \prime^{\prime} 02\left(0 .{ }^{\prime} 16\right)$ and $-0 .^{\prime} 06(0.12)$ for alpha and delta respectively. Therefore they are not different from the 2001 results presented in this paper, for which the equivalent statistics for 191 frames of Amalthea are - 0. '05 (0.'08) for alpha and $0.00\left(0 .{ }^{\prime} 06\right)$ for delta. For the 188 observations of Thebe, they are $-0{ }^{\prime} 03\left(0 .{ }^{\prime} 10\right)$ and $0{ }^{\prime} 03\left(0{ }^{\prime} 10\right)$, respectively for alpha and delta.

The results for 1995 and 1996 showed large residuals. Unfortunately the observations were made with small CCDs and in nights in which the images were of poor quality. However, the new reduction is much better than that used in the 1996 paper, bringing a significant improvement to the satellites positions.

Acknowledgements. The authors thank O. L. Chaves and R. Campos for their help with the observations, F. Vachier for the discussions about the observations of the inner satellites of Jupiter and A. H. Andrei for the discussions about the results. C.H.V. would like to thank the State of Rio de Janeiro Science Foundation-FAPERJ for providing computational facilities for this project and R.V.M. thank to CNPq and CAPES for the grants received.

\section{References}

Barnard, E. E. 1892, AJ, 12, 81

Colas, F. 1996, in Proc. of Phesat 95 Worshop, Supp. Ann. de Phys., 21,113

Kulyk, I., Jockers, K., Karpov, N., \& Sergeev, A. 2002, A\&A, 383, 724

Stone, E. C., \& Synnott, S. P. 1980, IAU Circ., 3470, 1

Synnott, S. P. 1980, Science, 210, 786

Vachier, F. 2004, Étude dynamique des satellites proches des planètes et des petites planètes, doctoral thesis, IMCCE-Observatoire de Paris

Veiga, C. H., \& Vieira Martins, R. 1996, Rev. Mex. Astron. Astrofis., Ser. Conf., 4, 128 